\title{
Outcome of High Grade Gliomas in Limited Resource Country (10 Years' Experience in Alexandria University Oncology Center 2003-2012)
}

\section{Mostafa K Barakat ${ }^{\star}$, Abdelaziz M Belal, Shadi H Fadel and Gamal H}

Department of Clinical Oncology and Nuclear Medicine, University of Alexandria, Egypt

"Corresponding author: Mostafa Kamal Barakat, Faculty of Medicine, Department Of Clinical Oncology and Nuclear Medicine, University of Alexandria, Egypt, Tel: 00201154522999; E-mail: mostafakamal2012.mk@gmail.com

Received date: Sep 09, 2016; Accepted date: Oct 05, 2016; Published date: Oct 15, 2016

Copyright: @ 2016 Barakat MK, et al. This is an open-access article distributed under the terms of the Creative Commons Attribution License, which permits unrestricted use, distribution, and reproduction in any medium, provided the original author and source are credited.

\begin{abstract}
Background: Gliomas are the most common form of malignant primary brain tumors in adults with an annual incidence of approximately five per 100,000 people. In Egypt, primary Central Nervous System (CNS) neoplasms are rare, constituting about $1-2 \%$ of all human neoplasms, with High Grade Gliomas (HGGs) being the most common type. The study aims to discover the histo-pathological aspect of HGGs, risk factors, and descriptive analysis of treatment received in Clinical oncology department affiliated with Alexandria University Hospital.
\end{abstract}

Methods: Data were collected and analyzed for four hundred and thirty four patients with histologically proven HGGs in the period between 2003 and 2012.

Results: Age of patients ranged from 21 to 83 years. Most cases (80.4\%) were Glioblastoma Multiform (GBM), followed by Anaplastic Astrocytoma (AA) reaching $14.3 \%$ of patients. In this study, $45.4 \%$ of patients had karnofsky performance score (KPS) more than $60 \%$. Only $2.5 \%$ of patients underwent gross total resection (GTR), $25.6 \%$ underwent Subtotal Resection (STR) and $50 \%$ of patients underwent excisional biopsy. Patients received radiotherapy were 355 about $81.8 \%$ of patients with HGGs $(80.2 \%$ of them received Radiotherapy (RT) only, $11.8 \%$ received RT followed by Adjuvant Chemotherapy (ACT), $4 \%$ received Concomitant Chemoradiotherapy (CCRT) and $4 \%$ received CCRT followed by ACT), $16.6 \%$ of patients received only best supportive care (BSC) and $2.3 \%$ did not receive treatment. The median Overall Survival (OS) was 10,13,19 and 15 months for patients received RT only, CCRT, CCRT followed by ACT and RT followed by ACT, respectively.

Conclusion: Delayed presentation and inadequate GTR together with unavailability of TMZ could affect outcome in limited resource countries.

Keywords: High grade gliomas; Glioblastoma multiform; Extent of surgical resection; Prognosis; Chemoradiotherapy; Temozolamide

\section{Introduction}

In Egypt, primary Central Nervous System (CNS) neoplasms are rare, consisting $1-2 \%$ of all human neoplasms with high grade gliomas (HGGs) being the most common type [1]. The average age of patients at the time of diagnosis is 64 years in the case of Glioblastomas (GBM) and 45 years in the case of Anaplastic Astrocytomas (AA) [2]. The most important prognostic factors in patients with HGGs are: age, tumor histology, Karnofsky Performance Status (KPS) and the extent of tumor resection [3]. Extent of resection and timing of radiotherapy affects the disease of outcome in limited resource country. The availability of specialized neuro-oncology surgeon and the availability of radiotherapy machines could affect the disease outcome [4,5].

\section{Patients and Methods}

We retrospectively reviewed the records of 434 consecutive patients with pathologically proven HGGs presented to the Department of clinical oncology and nuclear medicine, faculty of medicine, Alexandria University between 2003 and 2012 after approval of the ethics committee affiliated with Alexandria University.
The clinical features that were evaluated include: Age at time of registration, sex, performance status using Karnofsky Performance Status (KPS), neurological manifestations produced by the tumor mass, the adjacent edema, or the infiltration and destruction of normal tissue, radiological features of tumor either by CT or MRI which include presence of brain edema, mass effect and midline shift and histological type and grade were retrieved from the pathological reports of either excisional biopsy, Subtotal Resection (STR) or Gross Total Resection (GTR) and recorded as exactly present in original pathological report, which use the 2007 WHO classification of tumors of the central nervous system. Patients didn't undergo either excisional biopsy, STR or GTR were excluded from the study.

Treatment received was analysed, which includes:

- Radiotherapy received was recorded; patients were categorized as had radiotherapy dose $\geq 60$ Gy or dose $<60$ Gy and then categorized according to the intent of radiotherapy as definitive or palliative. Time of starting radiotherapy, radiotherapy duration, fractionation schedules and radiotherapy toxicity were recorded.

- Chemotherapy received was recorded; patients were categorized according to chemotherapy regimen received, number of chemotherapy cycles and toxicity of chemotherapy.

- Patients received best supportive care only was recorded and they were categorized as receiving BSC or not. 
Citation: Barakat MK, Belal AM, Fadel SH, Gamal H (2016) Outcome of High Grade Gliomas in Limited Resource Country (10 Years' Experience in Alexandria University Oncology Center 2003-2012). J Brain Tumors Neurooncol 1: 111. doi:10.4172/2475-3203.1000111

Page 2 of 9

Response to radiotherapy was categorized to complete response, regressive, stationary, and progression with progression is defined according to the modified WHO criteria as an increase in tumor size by $25 \%$, the appearance of new lesions, or an increased need for corticosteroids. The progression free survival period measured as the interval between the end of treatment and relapse or death or date of the last follow-up evaluation in patients who had no relapse and was estimated by Kaplan-Meier method.

We studied the impact of different clinical, pathological variables on survival using log rank test to compare between two or more estimated survival curves with $\mathrm{p}$ value $<0.05$ consider significant. For identification of factors that independently affected survival we used Cox proportional-hazard model-225 a minimum significance level of 0.05 on univariate analysis was used as criterion for determining multivariate testing. Statistical analysis was performed using IBM SPSS software package version 20.0.

\section{Results}

Four hundred and thirty four cases with histological proven HGGs were studied between 2003 and 2012, and the data were collected and analysed.

During this period, the incidence rate of HGGs ranged from $0.7 \%$ to $4.2 \%$ in our institute with increasing incidence (Figure 1). The age of patients ranged from 21 to 83 years and the mean age was 50 years. In this study, the number of male patients exceeds the number of female patients - namely 261 and 173 respectively, with male to female ratio of 1.5:1 (Table 1).

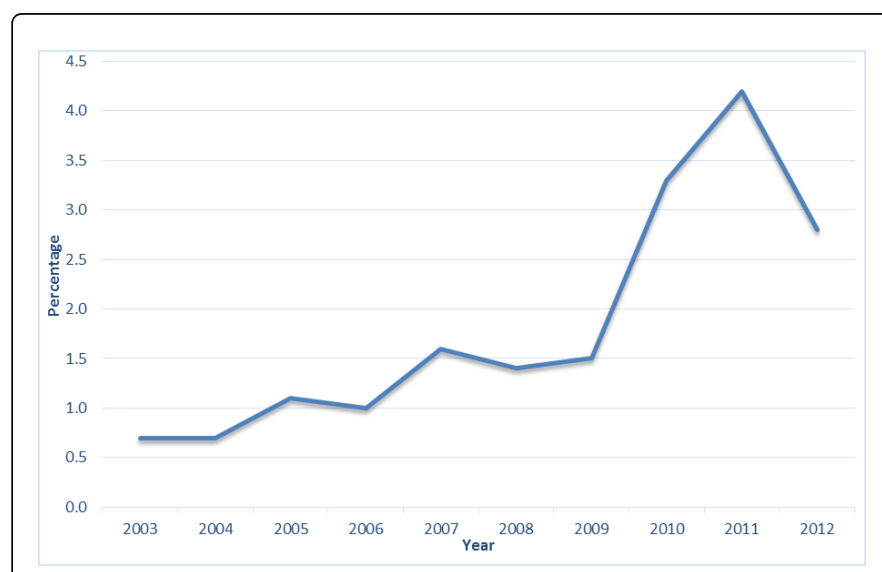

Figure 1: Descriptive chart for incidence of HGGs

According to KPS, 88 patients had KPS of $\leq 60 \%$ representing $20.3 \%$ of patients, while 197 patients (45.4\%) had KPS of $>60 \%$ (Table 1 ).

\begin{tabular}{|c|c|c|}
\hline & No. & $\%$ \\
\hline \multicolumn{3}{|l|}{ Sex } \\
\hline Female & 173 & 39.9 \\
\hline Male & 261 & 60.1 \\
\hline \multicolumn{3}{|l|}{ Age } \\
\hline$<50$ & 200 & 46.1 \\
\hline$\geq 50$ & 217 & 50 \\
\hline NA & 17 & 3.9 \\
\hline Median & 50 & \\
\hline \multicolumn{3}{|l|}{ Complain } \\
\hline Headache & 241 & 55.5 \\
\hline Blurred vision & 80 & 18.4 \\
\hline Convulsions & 54 & 12.4 \\
\hline Dizziness & 21 & 4.8 \\
\hline Vomiting & 47 & 10.8 \\
\hline Disturbed level of consciousness & 45 & 10.4 \\
\hline Motor deficit & 109 & 25.1 \\
\hline NA & 113 & 26 \\
\hline \multicolumn{3}{|l|}{ KPS } \\
\hline$>60 \%$ & 197 & 45.4 \\
\hline$\leq 60 \%$ & 88 & 20.3 \\
\hline
\end{tabular}


Citation: Barakat MK, Belal AM, Fadel SH, Gamal H (2016) Outcome of High Grade Gliomas in Limited Resource Country (10 Years' Experience in Alexandria University Oncology Center 2003-2012). J Brain Tumors Neurooncol 1: 111. doi:10.4172/2475-3203.1000111

Page 3 of 9

\begin{tabular}{|c|c|c|}
\hline NA & 149 & 34.3 \\
\hline Tumor Site & No. & $\%$ \\
\hline Cerebral & 327 & 75.3 \\
\hline Parietal & 158 & 48.3 \\
\hline Frontal & 100 & 30.6 \\
\hline Temporal & 37 & 11.3 \\
\hline Occipital & 14 & 4.3 \\
\hline Corpus callosum & 13 & 4 \\
\hline Whole hemisphere & 5 & 1.5 \\
\hline Cerebellar & 4 & 0.9 \\
\hline Thalamic & 7 & 1.6 \\
\hline NA & 96 & 22.1 \\
\hline \multicolumn{3}{|l|}{ Tumor volume } \\
\hline Min. - Max. & \multicolumn{2}{|l|}{$1.50-504.0$} \\
\hline Mean \pm SD & \multicolumn{2}{|l|}{$116.90 \pm 91.22$} \\
\hline Median & \multicolumn{2}{|l|}{97} \\
\hline \multicolumn{3}{|l|}{ Surgery } \\
\hline Biopsy & 217 & 50 \\
\hline STR & 111 & 25.6 \\
\hline GTR & 11 & 2.5 \\
\hline NA & 95 & 21.9 \\
\hline \multicolumn{3}{|l|}{ Histology } \\
\hline GBM & 348 & 80.2 \\
\hline AA & 62 & 14.3 \\
\hline $\mathrm{AOA}$ & 6 & 1.4 \\
\hline AOD & 13 & 3 \\
\hline $\mathrm{AE}$ & 5 & 1.2 \\
\hline \multicolumn{3}{|l|}{ Grade } \\
\hline IV & 348 & 80.2 \\
\hline III & 86 & 19.8 \\
\hline
\end{tabular}

Table 1: Clinic-pathological parameters of studied patients

With regards to histology, 348 patients $(80.2 \%)$ were presented with GBM, 62 patients (14.3\%) presented with AA, 6 patients (1.4\%) presented with Anaplastic Oligoastrocytoma (AOA). Anaplastic Oligodendroglioma (AOG) constituted 13 patients (3\%) while Anaplastic Ependymoma (AE) was found in 5 patients (1.2\%). In this study, 237 patients $(77.5 \%)$ presented with headache, 83 patients (27.1\%) presented with blurred vision, 65 patients $(21.2 \%)$ presented with neurological motor deficits, 52 patients $(17 \%)$ presented with convulsions, 49 patients (16\%) presented with disturbed level of consciousness, 41 patients (13.4\%) presented with vomiting, and 4 patients (1.3\%) presented with dizziness (Table 1 ).

Regarding diagnosis modalities, 58 patients (13.4\%) did a CT scan while 270 patients $(62.2 \%)$ did a MRI scan. We found that 327 patients $(96.7 \%)$ had their tumor in the cerebrum (158 patients of them $(48.3 \%)$ in parietal lobe, 100 patients $(30.6 \%)$ in frontal lobe, 37 patients (11.3\%) in temporal lobe, 14 patients (4.3\%) in occipital lobe, 13 patients $(4 \%)$ in corpus callosum, and 5 patients (1.5\%) in whole 
Citation: Barakat MK, Belal AM, Fadel SH, Gamal H (2016) Outcome of High Grade Gliomas in Limited Resource Country (10 Years' Experience in Alexandria University Oncology Center 2003-2012). J Brain Tumors Neurooncol 1: 111. doi:10.4172/2475-3203.1000111

Page 4 of 9

cerebral hemisphere), while 4 patients $(0.9 \%)$ had their tumor in the cerebellum and 7 patients (1.6\%) had their tumor in the thalamic area. As regards the extent of surgical excision, 11 patients $(2.5 \%)$ achieved GTR whereas 111 patients (25.6\%) achieved STR. Patients who had only tumor biopsy were 217 patients (50\%) (Table 1 ).

Most of our patients $(81.8 \%)$ received RT $(80.2 \%$ of them received RT only, $11.8 \%$ received RT followed by ACT, $4 \%$ received CCRT and $4 \%$ received CCRT followed by ACT), in addition to $16.6 \%$ of patients who received only BSC and 2.3\% did not receive treatment (Table 2).

Among patients received RT, 154 patients (52.4\%) received RT dose $\geq 60$ gray (Gy) and 63 patients (17.9\%) received RT dose $<60$ Gy. Eighty seven patients $(24.8 \%)$ received $<90 \%$ of planned RT dose. As regards to fractionation, 321 patients $(91.5 \%)$ received RT by conventional fractionation and 11 patients (3.1\%) received RT by hypofractionation. The median time from diagnosis till start of radiotherapy was 5.9 weeks (Table 3).

\begin{tabular}{|l|l|l|}
\hline Treatment & No. & $\%$ \\
\hline Radiotherapy & 355 & 81.8 \\
\hline RT only & 285 & 65.7 \\
\hline CCRTX & 14 & 3.2 \\
\hline CCRTX+ACT & 14 & 3.2 \\
\hline RT+ACT & 42 & 9.7 \\
\hline BSC & 72 & 16.6 \\
\hline No treatment & 10 & 2.3 \\
\hline
\end{tabular}

Table 2: Descriptive the studied cases according treatment modalities

\begin{tabular}{|c|c|c|}
\hline & No. & $\%$ \\
\hline \multicolumn{3}{|l|}{ Radiotherapy } \\
\hline Yes & 355 & 81.8 \\
\hline No & 79 & 18.2 \\
\hline \multicolumn{3}{|c|}{ Intent of treatment $(n=355)$} \\
\hline Definitive & 341 & 96.1 \\
\hline Palliative & 14 & 3.9 \\
\hline \multicolumn{3}{|l|}{ Dose $(n=355)$} \\
\hline$\geq 60 \mathrm{~Gy}$ & 188 & 53 \\
\hline$>60 \mathrm{~Gy}$ & 63 & 17.7 \\
\hline$<90 \%$ of paned does & 87 & 24.5 \\
\hline NA & 17 & 4.8 \\
\hline \multicolumn{3}{|l|}{ Fractionation $(n=355)$} \\
\hline Conventional & 325 & 91.5 \\
\hline Hypofractionation & 11 & 3.1 \\
\hline NA & 19 & 5.4 \\
\hline \multicolumn{3}{|c|}{ Radiotherapy response $(n=108)$} \\
\hline Stationary & 76 & 70.4 \\
\hline Regressive & 12 & 11.1 \\
\hline Progressive & 18 & 16.7 \\
\hline Complete Response & 2 & 1.8 \\
\hline NA & 247 & 69.6 \\
\hline \multicolumn{3}{|c|}{ Duration till response $(n=90)$} \\
\hline Min.-Max. & $15.0-120.0$ & \\
\hline Mean \pm SD & $46.54 \pm 22.53$ & \\
\hline Median & 42 & \\
\hline
\end{tabular}


Citation: Barakat MK, Belal AM, Fadel SH, Gamal H (2016) Outcome of High Grade Gliomas in Limited Resource Country (10 Years' Experience in Alexandria University Oncology Center 2003-2012). J Brain Tumors Neurooncol 1: 111. doi:10.4172/2475-3203.1000111

Page 5 of 9

\begin{tabular}{|l|l|l|}
\hline Radiotherapy Toxicity $(\mathbf{n}=\mathbf{3 5 5})$ & & \\
\hline Headache & 48 & 13.5 \\
\hline Blurring of vision & 7 & 2 \\
\hline Vomiting & 8 & 2.3 \\
\hline Fatigue & 1 & 0.3 \\
\hline Convulsions & 3 & 0.8 \\
\hline Gastritis & 5 & 1.4 \\
\hline Disorientation & 5 & 1.4 \\
\hline NA & 305 & 85.9 \\
\hline
\end{tabular}

Table 3: Descriptive the studied cases according radiotherapy

In this study series, $72(16.6 \%)$ patients received chemotherapy. Temozolamide (TMZ) was used in $65.3 \%$ of those patients, while $1.6 \%$ of them received PCV (Procarbazine Carmustine and Vincristine).
And the rest of them received other treatment protocols. The average number of chemotherapy cycles was 6 (Table 4 ).

\begin{tabular}{|c|c|c|}
\hline & No. & $\%$ \\
\hline \multicolumn{3}{|l|}{ Chemotherapy } \\
\hline Yes & 72 & 16.6 \\
\hline No & 362 & 83.4 \\
\hline \multicolumn{3}{|l|}{ Protocol $(n=72)$} \\
\hline TMZ & 47 & 65.3 \\
\hline PCV & 7 & 1.6 \\
\hline Carboplatin-Etoposide & 4 & 5.6 \\
\hline Cisplatin-Etoposide & 12 & 16.7 \\
\hline Vincristine-Dacarbazine & 1 & 1.4 \\
\hline Oral Etoposide & 4 & 5.6 \\
\hline Cisplatin-Irinotecan-TMZ & 1 & 1.4 \\
\hline NA & 2 & 2.8 \\
\hline \multicolumn{3}{|l|}{ Intent $(n=72)$} \\
\hline Adjuvant & 42 & 58.3 \\
\hline Concomitant & 14 & 19.4 \\
\hline Concomitant and adjuvant & 14 & 19.4 \\
\hline Palliative & 2 & 2.8 \\
\hline \multicolumn{3}{|l|}{ Number of cycles $(n=72)$} \\
\hline NA & 34 & 47.2 \\
\hline Min.-Max. & \multicolumn{2}{|l|}{$3.0-18.0$} \\
\hline Mean \pm SD & \multicolumn{2}{|l|}{$6.68 \pm 3.65$} \\
\hline Median & \multicolumn{2}{|l|}{6} \\
\hline
\end{tabular}


Citation: Barakat MK, Belal AM, Fadel SH, Gamal H (2016) Outcome of High Grade Gliomas in Limited Resource Country (10 Years' Experience in Alexandria University Oncology Center 2003-2012). J Brain Tumors Neurooncol 1: 111. doi:10.4172/2475-3203.1000111

Page 6 of 9

\begin{tabular}{|l|l|l|}
\hline \multicolumn{2}{|l|}{} & \multicolumn{2}{|l|}{} & \\
\hline Response to chemotherapy $(\mathbf{n}=\mathbf{7 2})$ & & 66.7 \\
\hline NA & 48 & 20.8 \\
\hline Stationary & 15 & 6.9 \\
\hline Regressive & 5 & 5.6 \\
\hline Progressive & 4 & 0 \\
\hline Complete Response & 0 & \\
\hline
\end{tabular}

Table 4: Descriptive the studied cases according chemotherapy

Treatment response was assessed only in 108 patients with $1.8 \%$ were disease free, $70.4 \%$ had a stationary course, $16.7 \%$ had a progressive course, and finally $11.1 \%$ had a regressive course.

Treatment response after RT was reported only in 108 patients. Among patients that underwent GTR $(\mathrm{n}=10)$, treatment response was reported in 8 patients who achieved stationary course where 26 patients $(28.9 \%)$ of patients underwent STR $(n=122)$ achieved stationary course, 2 patients $(2.2 \%)$ achieved disease free, 5 patients $(5.6 \%)$ achieved regressive course and 7 patients (7.8\%) developed progression. Of all the patients that underwent excisional biopsy $(n=175), 35$ patients $(20 \%)$ achieved stationary course, 7 patients $(4 \%)$ achieved regressive course, 11 patients $(6.3 \%)$ developed progression, and no patients developed disease free (Table 5).

Among patients who received RT only $(\mathrm{n}=285), 58$ patients $(20.4 \%)$ achieved stationary course, 7 patients (2.5\%) achieved regressive course while 12 patients $(4.2 \%)$ developed progression and only 1 patient $(0.3 \%)$ achieved disease free.

\begin{tabular}{|c|c|c|c|c|c|c|c|c|c|c|c|c|}
\hline \multirow{3}{*}{ Surgery } & \multicolumn{12}{|c|}{ Response of radiotherapy } \\
\hline & \multicolumn{2}{|l|}{ NA } & \multicolumn{2}{|c|}{ Stationary } & \multicolumn{2}{|c|}{ Regressive } & \multicolumn{2}{|c|}{ Progression } & \multicolumn{2}{|c|}{ Disease free } & \multicolumn{2}{|c|}{ Total } \\
\hline & No & $\%$ & No & $\%$ & No & $\%$ & No & $\%$ & No & $\%$ & No & $\%$ \\
\hline Biopsy & 122 & 69.7 & 35 & 20 & 7 & 4 & 11 & 6.3 & 0 & 0 & 175 & 100 \\
\hline STR & 50 & 55.5 & 26 & 28.9 & 5 & 5.6 & 7 & 7.8 & 2 & 2.2 & 90 & 100 \\
\hline GTR & 2 & 20 & 8 & 80 & 0 & 0 & 0 & 0 & 0 & 0 & 10 & 100 \\
\hline
\end{tabular}

Table 5: Response to treatment after radiotherapy in relation to surgery

Among patients that received RT followed by ACT were 42 patients with 12 patients (28.6\%) achieved stationary course, 4 patients $(9.5 \%)$ achieved regressive course and 5 patients (11.9\%) developed progression. Whereas 14 patients received CCRT, treatment response was reported only in 2 patients (14.3\%) who achieved stationary response. Fourteen patients received CCRT followed by ACT, with 4 patients (28.7\%) achieved stationary course, 1 patient (7.1\%) achieved regressive course, 1 patient (7.1\%) achieved progressive course and one patient $(7.1 \%)$ was free (Table 6). The median OS of all studied patients was 9 months (Figure 2) and the median Progression Free Survival (PFS) was 8 months (Figure 3). The median OS was 10, 13, 19 and 15 months for patients received RT only, CCRT, CCRT followed by ACT and RT followed by ACT, respectively (Figure 4).

\begin{tabular}{|c|c|c|c|c|c|c|c|c|c|c|c|c|}
\hline \multirow{3}{*}{ Treatment } & \multicolumn{12}{|c|}{ Response of radiotherapy } \\
\hline & \multicolumn{2}{|l|}{ NA } & \multicolumn{2}{|c|}{ Stationary } & \multicolumn{2}{|c|}{ Regressive } & \multicolumn{2}{|c|}{ Progression } & \multicolumn{2}{|c|}{ Disease free } & \multicolumn{2}{|c|}{ Total } \\
\hline & No & $\%$ & No & $\%$ & No & $\%$ & No & $\%$ & No & $\%$ & No & $\%$ \\
\hline $\mathrm{RT}+\mathrm{ACT}$ & 21 & 50 & 12 & 28.6 & 4 & 9.5 & 5 & 11.9 & 0 & 0 & 42 & 100 \\
\hline CCRTX & 12 & 85.7 & 2 & 14.3 & 0 & 0 & 0 & 0 & 0 & 0 & 14 & 100 \\
\hline CCRTX+ACT & 7 & 50 & 4 & 28.7 & 1 & 7.1 & 1 & 7.1 & 1 & 7.1 & 14 & 100 \\
\hline RT only & 207 & 72.6 & 58 & 20.4 & 7 & 2.5 & 12 & 4.2 & 1 & 0.3 & 285 & 100 \\
\hline
\end{tabular}

Table 6: Treatment response after radiotherapy 
Citation: Barakat MK, Belal AM, Fadel SH, Gamal H (2016) Outcome of High Grade Gliomas in Limited Resource Country (10 Years' Experience in Alexandria University Oncology Center 2003-2012). J Brain Tumors Neurooncol 1: 111. doi:10.4172/2475-3203.1000111

Page 7 of 9

\section{Discussion}

Fifty percent of patients were above 50 years old, close to the number reported by Aboziada et al., in Egypt (48.1\%) [6]. However, data from western countries were much different. Stupp et al. reported that $67 \%$ of patients are above the age of 50s [7]. And this could be explained by longer expected age in Developed countries if compared with that in developing countries due to the presence of infectious diseases and poor health management which results in shorter expected age. So, it draws attention to a very important different clinicpathological parameter between two populations that would affect the choice of treatment.

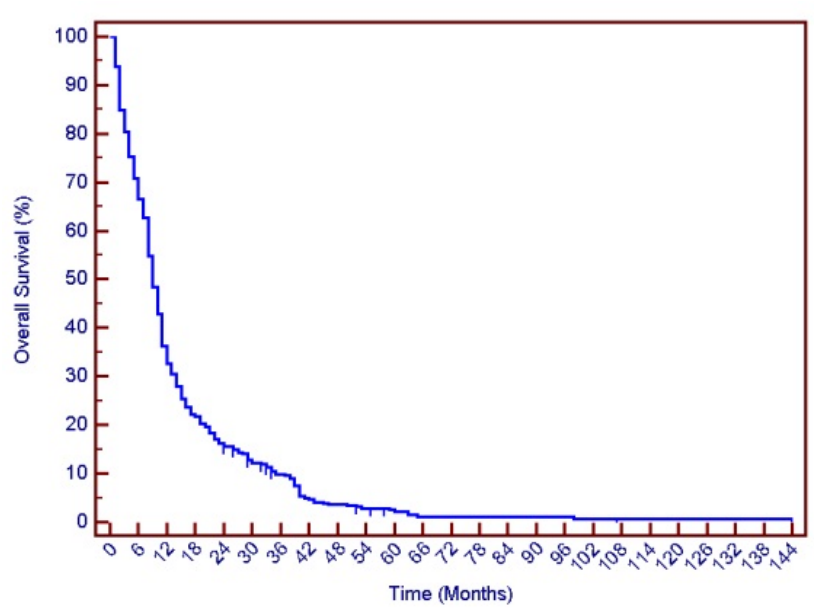

Figure 2: Kaplan-Meier survival curve for overall survival

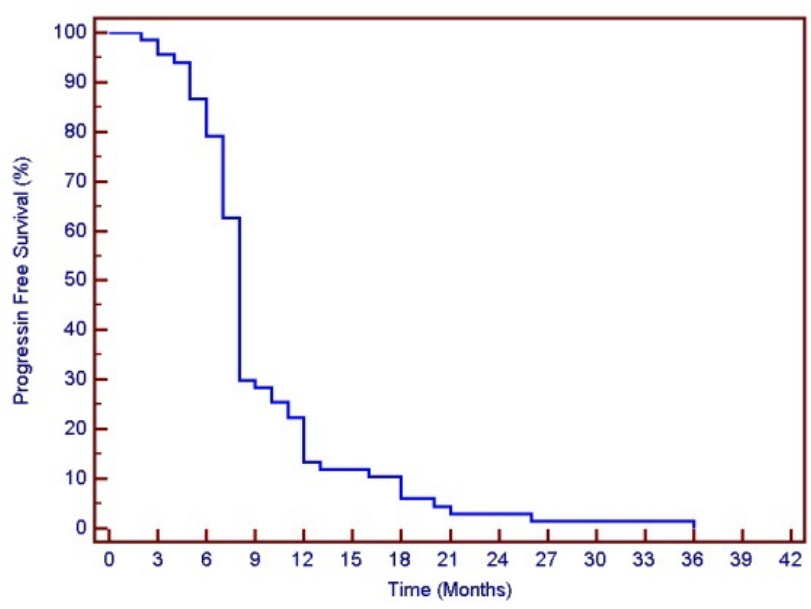

Figure 3: Kaplan-Meier survival curve for progression free survival

In this study, there is a significant association between age at presentation ( $\leq 50 \mathrm{ys}$ vs $>50 \mathrm{ys}$ ) and OS ( $\mathrm{p}$ value $<0.001$ ) (Figure 5$)$. As a result, the age at presentation was an important predictor of the outcome of patients and this is consistent with data reported in either Egyptian or western countries studies [6-9].

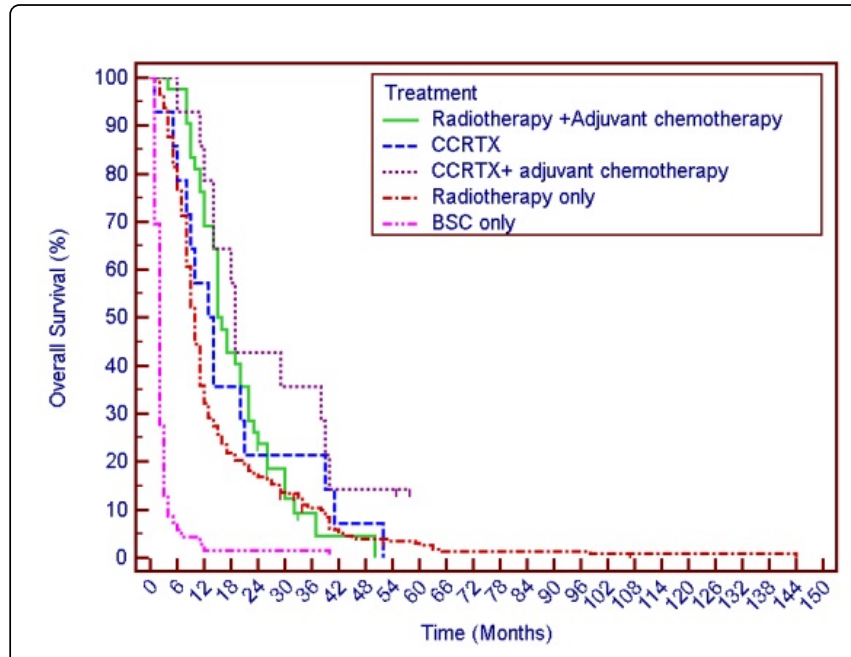

Figure 4: Kaplan-Meier survival curve for overall survival with treatment

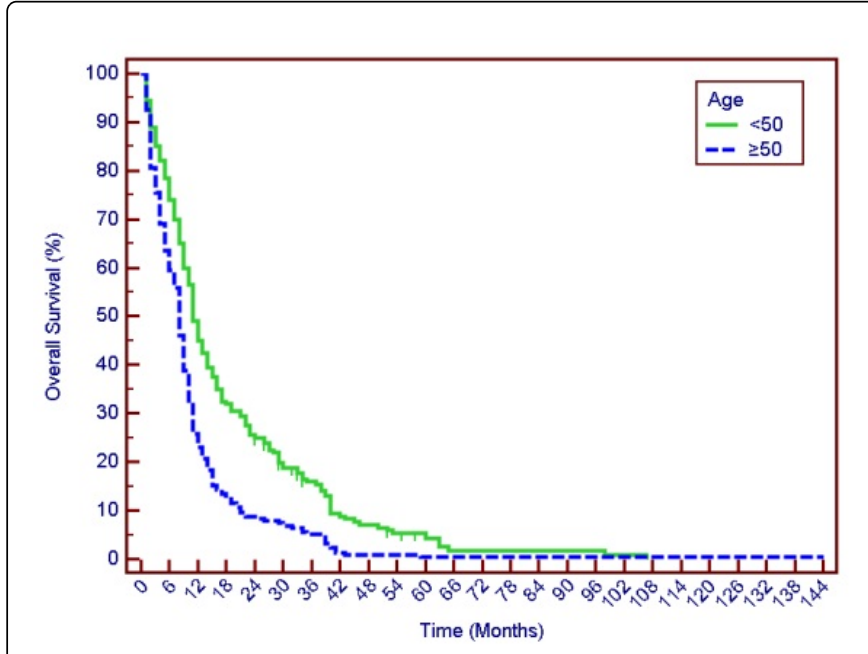

Figure 5: Kaplan-Meier survival curve for overall survival with age

Tezcan et al. reported that $52 \%$ of patients had KPS more than $60 \%$ which was nearly similar to our results reaching $45.4 \%$ [10]. In this study, there was a significant positive association between KPS and improving treatment outcome and OS $(\mathrm{P}$ value $<0.001)$ and this coincides with what reported by many studies in literature (Figure 6) [11-16].

GBM comprises the most common histological subtype of HGGs in our patients $(80.2 \%)$ which coincides with what reported by Irwin et al. [15]. GBM had the worst median OS (8 months) similar to the results reported by Yilmaz et al. [9].

As regards surgical resection, only $2.5 \%$ of studied patients underwent GTR, 25.6\% underwent STR while the rest of studied patients (50\%) underwent excisional biopsy and this coincides with what reported by Mohan et al , where 7\% underwent GTR , $40 \%$ underwent STR and 53\% underwent excisional biopsy [16]. However, this was much different from what was reported by most other series in 
the literature where most of cases underwent GTR. This may be due to delayed presentation of patients in our center which causes difficulties to our neurosurgeons to do GTR and so therefore leaves a negative significant impact on overall survival in comparison to western countries where adequate surgical resection leads to a more favorable survival $[9,17]$.

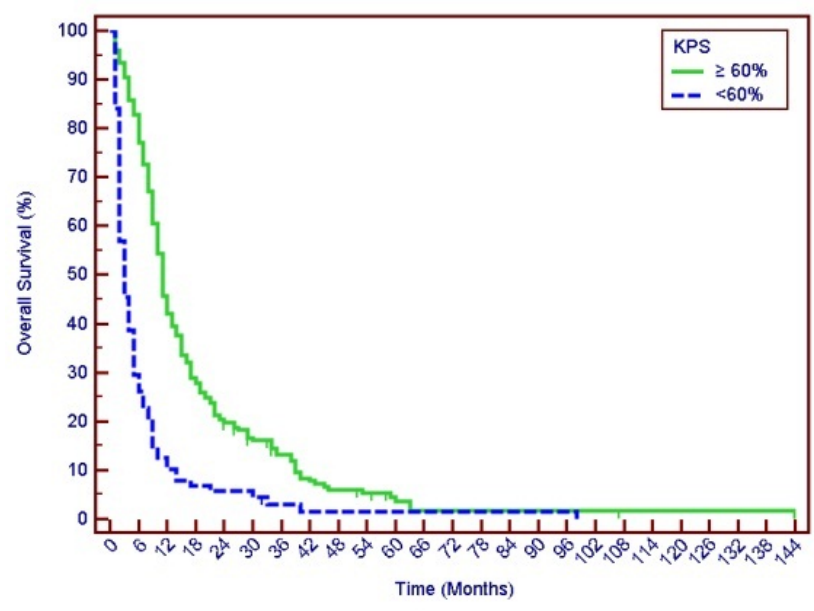

Figure 6: Kaplan-Meier survival curve for overall survival with KPS

Nitta et al., Albert et al. and Olive et al. reported that extent of surgical resection had a significant association with OS. This study reported the same results in which the median OS was 21, 11 and 9 months for GTR, STR and excisional biopsy respectively [18-20].

Among our studied patients, 355 patients (81.8\%) received adjuvant RT, $10(2.3 \%)$ patients did not receive any treatment and $72(16.6 \%)$ patients received only Best Supportive Care (BSC) and this coincides with what reported by Chang et al., where most of the patients (89\%) received RT only with no systemic treatment [21]. This could be explained by limited resources in our country and shortage of chemotherapeutic agents (e.g. TMZ) due to cost issues.

In our study we found that average time from diagnosis till start of RT was 5.9 weeks. This is similar to what was reported by Stupp et al., where average time of diagnosis was 5 weeks [22] and also compared to what was reported by Adeberg et al., where the average time from diagnosis to start RT was 35 days [5].

We did not find a significant association between delay in RT and PFS and OS with $\mathrm{p}$ value 0.313 and 0.323 respectively and this coincides with what was reported by Adeberg et al. [5] but much different from what was reported by Irwin et al. [15]. These unexpected findings might be explained by large tumor burden due to inadequate surgical resection which may affect outcome of patients in our center rather than delay of starting radiotherapy.

In this study series, only $72(16.6 \%)$ patients received chemotherapy; forty two of them received ACT; 14 patients received concomitant CCRT and 14 patients received CCRT followed by ACT. This is different from what has been reported in most of series in the literature where most patients received concomitant and adjuvant chemotherapy. This may have been caused by unavailability of TMZ in our center due to cost issues [17,22].
As regards chemotherapy protocols received, we found that $65.3 \%$ of patients received chemotherapy received TMZ, either concomitant or adjuvant, which coincides with the standard of care in most of series in the literature $[17,22]$. Furthermore, the median number of ACT cycles was 6 cycles that was similar to the results of stupp et al. and Yilmez et al. $[7,9]$.

In this study, patients received CCRT had improved OS but not significantly ( $\mathrm{p}$ value 0.464 ) in contrast to what was reported by other studies in the literature. This could be explained by limited usage of TMZ in our center due to logistic and cost issues. As a result, only small number of studied patients received concomitant TMZ [22-25].

We also found that patients received ACT had a significant impact on OS, with median OS of 15 months versus 10 months in patients received RT only, and this coincides with what was reported by Ekici et al. [26].

In comparison to patients received RT only, patients received CCRT followed by ACT had more favorable OS (19 months Vs 10 months) with $\mathrm{p}$ value 0.008 . Graus et al. reported the same results with median OS of 16.5 months and 7.8 months for those received CCRTx followed by adjuvant TMZ and RT alone respectively [17].

\section{Recommendations}

Inadequate tumor resection and shortage of chemotherapeutic agents negatively affect outcome of patients with HGGs in our country so we recommend that management of patients with HGGs should be highly individualized and should take a multidisciplinary approach involving neuro-oncology, neurosurgery, radiation oncology, and pathology, to optimize treatment outcomes.

\section{Conclusion}

Delayed presentation and inadequate GTR together with unavailability of TMZ could affect outcome in limited resource countries.

\section{References}

1. Ibrahim AS, Khaled HM, Mikhail NNH, Baraka H, Kamel H (2014) Cancer Incidence in Egypt: Results of the National Population-Based Cancer Registry Program. JCancer Epidemiol 2014: 437971.

2. Dolecek TA, Propp JM, Stroup NE, Kruchko C (2012) CBTRUS statistical report: primary brain and central nervous system tumors diagnosed in the United States in 2005-2009. Neuro Oncol 14: v1-49.

3. Li J, Wang M, Won M, Shaw EG, Coughlin C, et al. (2011) Validation and simplification of the Radiation Therapy Oncology Group recursive partitioning analysis classification for glioblastoma. Int J Radiat Oncol Biol Phys 81: 623-630.

4. Lacroix M, Abi-Said D, Fourney DR, Gokaslan ZL, Shi W, et al. (2001) A multivariate analysis of 416 patients with glioblastoma multiforme: prognosis, extent of resection, and survival. J Neurosurg 95: 190-198.

5. Adeberg S, Bostel T, Harrabi S, Bernhardt D, Welzel T, et al. (2015) Impact of delays in initiating postoperative chemoradiation while determining the MGMT promoter-methylation statuses of patients with primary glioblastoma. BMC cancer 15: 1 .

6. Aboziada MA, Abo-Kresha AE (2012) Hypofractionated conformal irradiation of patients with malignant glioma. J Egypt Natl Canc Inst 24: 139-143.

7. Stupp R, Hegi ME, Mason WP, van den Bent MJ, Taphoorn MJ, et al. (2009) Effects of radiotherapy with concomitant and adjuvant temozolomide versus radiotherapy alone on survival in glioblastoma in a 
Citation: Barakat MK, Belal AM, Fadel SH, Gamal H (2016) Outcome of High Grade Gliomas in Limited Resource Country (10 Years' Experience in Alexandria University Oncology Center 2003-2012). J Brain Tumors Neurooncol 1: 111. doi:10.4172/2475-3203.1000111

Page 9 of 9

randomised phase III study: 5-year analysis of the EORTC-NCIC trial Lancet Oncol 10: 459-466.

8. Gaber M, Salim H, El-Nahas T (2013) Prospective study evaluating the radiosensitizing effect of reduced doses of temozolomide in the treatment of Egyptian patients with glioblastoma multiforme. Cancer Manag Res 5: 349-356.

9. Yilmaz ER, Gürer B, Kertmen H, Dolgun H, Sanli AM, et al. (2011) The Outcome of Surgically Resected Anaplastic Astrocytoma and Glioblastoma: Results of Single Center Retrospective Study. Journal of Neurological Sciences 28: 347-354.

10. Tezcan Y, Koc M (2011) 3-D conformal radiotherapy with concomitant and adjuvant temozolomide for patients with glioblastoma multiforme and evaluation of prognostic factors. Radiol Oncol 45: 213-219.

11. Dehdashti AR, Sharma S, Laperriere N, Bernstein M (2007) Coincidence vs cause: cure in three glioblastoma patients treated with brachytherapy. Can J Neurol Sci 34: 339-342.

12. Krex D, Klink B, Hartmann C, von Deimling A, Pietsch T, et al. (2007) Long-term survival with glioblastoma multiforme. Brain 130: 2596-2606.

13. Ulutin C, Fayda M, Aksu G, Cetinayak O, Kuzhan O, et al. (2006) Primary glioblastoma multiforme in younger patients: a single-institution experience. Tumori 92: 407-411.

14. Hung KS, Howng SL (2003) Prognostic significance of annexin VII expression in glioblastomas multiforme in humans. J Neurosurg 99: 886-892.

15. Irwin C, Hunn M, Purdie G, Hamilton D (2007) Delay in radiotherapy shortens survival in patients with high grade glioma. J Neurooncol 85 339-343.

16. Mohan DS, Suh JH, Phan JL, Kupelian PA, Cohen BH, et al. (1998) Outcome in elderly patients undergoing definitive surgery and radiation therapy for supratentorial glioblastoma multiforme at a tertiary care institution. Int J Radiat Oncol Biol Phys 42: 981-987.
17. Graus F, Bruna J, Pardo J, Escudero D, Vilas D, et al. (2013) Patterns of care and outcome for patients with glioblastoma diagnosed during 2008-2010 in Spain. Neuro Oncol 15: 797-805.

18. Nitta T, Sato K (1995) Prognostic implications of the extent of surgical resection in patients with intracranial malignant gliomas. Cancer 75 : 2727-2731.

19. Albert FK, Forsting M, Sartor K, Adams HP, Kunze S (1994) Early postoperative magnetic resonance imaging after resection of malignant glioma: objective evaluation of residual tumor and its influence on regrowth and prognosis. Neurosurgery 34: 45-61.

20. Olivi A, Blakeley J, Sills AK (2009) Current Controversies In The Management Of High Grade Gliomas: An Interactive Case Discussion.

21. Chang SM, Parney IF, Huang W, Anderson FA Jr, Asher AL, et al. (2005) Patterns of care for adults with newly diagnosed malignant glioma. JAMA 293: 557-564.

22. Stupp R, Mason WP, van den Bent MJ, Weller M, Fisher B, et al. (2005) Radiotherapy plus concomitant and adjuvant temozolomide for glioblastoma. N Engl J Med 352: 987-996.

23. Stewart LA (2002) Chemotherapy in adult high-grade glioma: a systematic review and meta-analysis of individual patient data from 12 randomised trials. Lancet 359: 1011-1018.

24. Fine HA, Dear KB, Loeffler JS, Black PM, Canellos GP (1993) Metaanalysis of radiation therapy with and without adjuvant chemotherapy for malignant gliomas in adults. Cancer 71: 2585-2597.

25. Athanassiou H, Synodinou M, Maragoudakis E, Paraskevaidis M, Verigos C, et al. (2005) Randomized phase II study of temozolomide and radiotherapy compared with radiotherapy alone in newly diagnosed glioblastoma multiforme. J Clin Oncol 23: 2372-2377.

26. Ekici MA, Bulut T, Tucer B, Basarslan SK, Kurtsoy A (2013) Prognostic factors in patients with glioblastoma multiforme (clinical research). Turk J Med Sci 43: 795-804. 